\title{
Fuzzy Kinematic Reliability of a Cartesian Parallel Manipulator with Clearances*
}

\author{
Fabian A. Lara-Molina ${ }^{1}$ and Didier Dumur ${ }^{2}$
}

\begin{abstract}
This paper presents a novel method to assess the kinematic reliability of parallel manipulators based on the fuzzy theory. For this purpose, the error propagation method permits to compute the position error in the endeffector, taking into account the clearances, and the kinematic constraints of the parallel manipulator. The failure possibility conveys an assessment of the insight kinematic performance that can not be obtained by the conventional methods used in the literature. Numerical results are compared with the well known probabilistic approach based on the Monte Carlo Simulation (MCS).
\end{abstract}

\section{INTRODUCTION}

Parallel mechanisms are unavoidably affected by uncertainties produced by manufacturing and assembly error of the links, backlash positioning error of the actuators, and joints clearances. The calibration of the mechanism reduces the effect of manufacturing and assembly errors of the links significantly. However, the errors produced by joint clearances can not be correctly compensated by the calibration methods [1]. Moreover, joint clearances are necessary for the relative motion between the links; therefore, they are the most important source of error that affects the accuracy and repeatability of the mechanisms [2]. For this reason, it is necessary to develop computational methods to analyze the effects of joint clearances in the pose error of mechanisms.

The joint clearances in passive joints of parallel mechanisms produce unconstrained end-effector motions when the active joints are blocked [3]. The clearances in the axisymmetrical joints were previously modeled by [4]. Moreover, the uncertainty effect of joint clearance has widely been studied by using probabilistic approaches [5]-[8].

Several approaches have been developed to assess the influence of joint clearances on the kinematic accuracy of mechanisms. The pose error of the links has been determined by using a kinematic method [9]. Moreover, the kinematic accuracy of parallel manipulators with joint clearances has been analyzed. The error analysis problem of parallel manipulators has been studied based on a standard convex optimization [4].

The kinematic reliability has also been studied to evaluate the effect of the clearances on the kinematic accuracy.

\footnotetext{
*This work was not supported by the CNPq-Process 427204/2018-6 and CAPES

${ }^{1}$ F. A. Lara-Molina is with the Federal University of Technology - Paraná, Cornélio Procópio, PR 86300-00 Brazil (e-mail: fabianmolina@utfpr.edu.br).

${ }^{2} \mathrm{D}$. Dumur is with the Laboratoire des Signaux et Systémes, CentraleSupélec-CNRS-Univ. Paris-Sud, Univ. Paris-Saclay, Control Department, 91 192, Gif sur Yvette cedex, France (e-mail: Didier.Dumur@centralesupelec.fr).
}

Approaches based on probabilistic theory have analyzed the sensitivity and reliability [10], the reliability in the entire trajectory based on the maximum entropy principle [5], and the time-dependent reliability [7]. As an alternative, non-probabilistic methods have recently proposed, such as convex models [11] and interval approaches [12]. The aforementioned approaches compute indices that measure the probability that the positioning error surpasses an admissible limit; nevertheless, these indices do not deliver a direct relationship with the magnitude of the error. Therefore, the present contribution proposes an alternative reliability index based on the fuzzy theory that permits to evaluate the possibility of error; the definition of this possibility of error is directly linked with the maximum positioning error produced by clearances, i.e. it expresses the possibility that error exceeds a determined admissible limit. This reliability index could be used as a performance criterion considered in the optimal design of robotic manipulators [13]. Moreover, the fuzzy theory has already been successfully applied to control of robotic manipulators [14]-[16].

This contribution presents a novel method to assess the kinematic reliability of a cartesian parallel manipulator based on the fuzzy theory. First, the clearance model with uncertain parameters is presented based on the axisymmetric joint model. Then, the error propagation method determines the pose error of the end-effector produced by the joint clearances considering the kinematic constraints of the Cartesian Parallel Manipulator (CPM). Finally, the kinematic reliability that expresses the possibility that error exceds a determined limit is computed. The proposed approach to evaluate the fuzzy kinematic reliability consists of three stages: $i$ ) the joint clearance model with fuzzy uncertainties; ii) the method to propagate the fuzzy error of the joints to compute the positioning error on the end-effector; iii) the fuzzy kinematic reliability evaluation based on the proposed possibility of failure.

\section{JOINT CLEARANCE MODEL}

Clearances introduce additional and uncontrollable degrees of freedom within the joints according to the axisymmetric joint clearance model that considers the joint axis along the $z$-axis (see Fig. 1). These additional degrees of freedom can be either rotational and translational; consequently, the pose error at the local frame of the joint can be modeled using the error screw $\delta \mathbf{e}_{i, j}$, thus:

$$
\delta \mathbf{e}_{i, j}=\left[\begin{array}{ll}
\delta \mathbf{r}_{i, j} & \delta \mathbf{t}_{i, j}
\end{array}\right]^{T}
$$


where $i$ is the index of the kinematic chain, and $j$ is the index of the joint in the respective $i^{\text {th }}$ kinematic chain, $\delta \mathbf{r}_{i, j}=\left[\begin{array}{lll}\delta r_{i, j, x} & \delta r_{i, j, y} & \delta r_{i, j, z}\end{array}\right]^{T}$ is the orientation error, and $\delta \mathbf{t}_{i, j}=\left[\begin{array}{lll}\delta t_{i, j, x} & \delta t_{i, j, y} & \delta t_{i, j, z}\end{array}\right]^{T}$ is the translational error produced by the clearances with respect to the local frame $F_{i, j}$ (see Fig. 1).
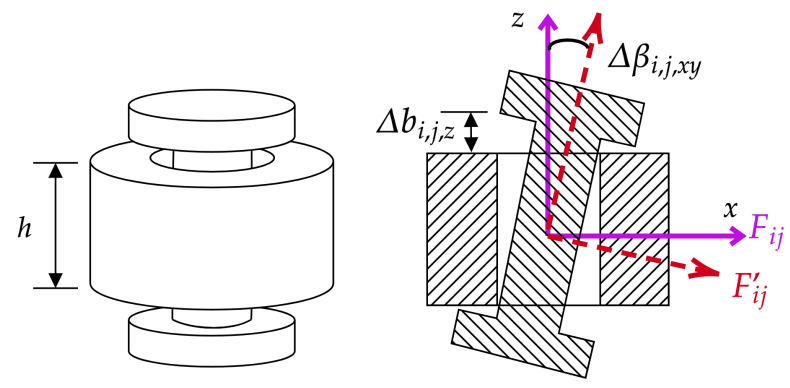

(a)

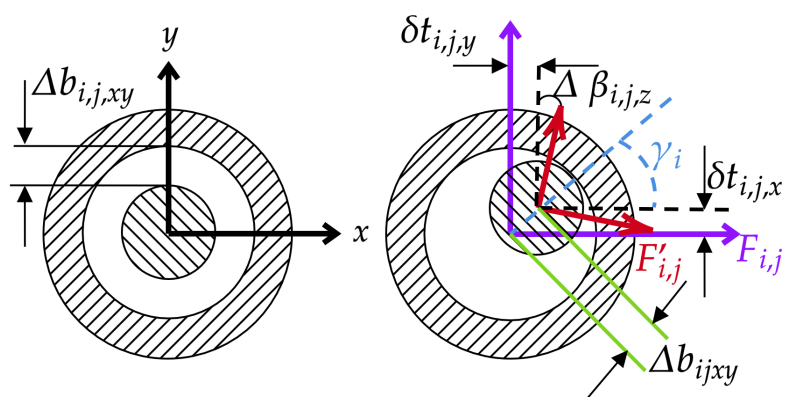

(b)

Fig. 1. Joint Clearance Model: (a) $x y$ plane, (b) $x y$ plane

The translational clearance along the axis joint $z$, and the rotational clearance with respect to the axis $z$ are defined as $\Delta b_{i, j, z}$ and $\Delta \beta_{i, j, x y}$, respectively. Moreover, the translational clearance in the $x y$ plane and the rotational clearance related to the $z$ axis are defined as $\Delta b_{i, j, x y}$ and $\Delta \beta_{i, j, z}$. Therefore, the elements of the error screw $\delta \mathbf{e}_{i, j}$ of Eq. (1) are defined by:

$$
\left\{\begin{array} { l } 
{ \delta r _ { i , j , x } = \Delta \beta _ { i , j , x y } \operatorname { c o s } ( \gamma _ { i } ) } \\
{ \delta r _ { i , j , y } = \Delta \beta _ { i , j , x y } \operatorname { s i n } ( \gamma _ { i } ) } \\
{ \delta r _ { i , j , z } = \Delta \beta _ { i , j , z } }
\end{array} \quad \left\{\begin{array}{l}
\delta t_{i, j x}=\Delta b_{i, j, x y} \cos \left(\gamma_{i}\right) \\
\delta t_{i, j y}=\Delta b_{i, j, x y} \sin \left(\gamma_{i}\right) \\
\delta t_{i, j z}=\Delta b_{i, j, z}
\end{array}\right.\right.
$$

with $0 \leq \gamma_{i} \leq 2 \pi$. Following this definition, the error pose should meet the following constraints: $\delta r_{i, j, x}^{2}+\delta r_{i, j, y}^{2} \leq$ $\Delta \beta_{i, j, x y}^{2}$ and $\delta t_{i, j, x}^{2}+\delta t_{i, j, y}^{2} \leq \Delta b_{i, j, x y}^{2}$. The uncertainties are introduced in the following five parameters that defines the clearances of the joints: $\Delta \beta_{i, j, z}, \Delta \beta_{i, j, x y}, \gamma_{i}, \Delta b_{i, j, x y}$ and $\Delta b_{i, j, z}$.

\section{ERror Propagation Method}

\section{A. Serial kinematic Chain}

Initially, the Denavit-Hartenberg method is used to obtain the pose of the end-effector considering no clearances. Thus, the homogeneous transformation matrix, $S_{i, j}$, is defined as:

$$
\mathbf{S}_{i, j}=\left[\begin{array}{cc}
\mathbf{R}_{i, j} & \mathbf{t}_{i, j} \\
\mathbf{0}_{1 \times 3} & 1
\end{array}\right]
$$

with $i=1, \ldots, m$ and $j=1, \ldots, n_{i, f}$, respectively; $m$ is the number of kinematic chains (for a single kinematic chain $m=1$ ), and $n_{i, f}$ is the total number of frames. $\mathbf{S}_{i, j}$ represents the transformation matrix from the frame $\mathbf{F}_{i, j}$ to the frame $\mathbf{F}_{i, j+1}, \mathbf{R}_{i, j}$ is the (3x3) rotation matrix and $\mathbf{t}_{i, j}$ translation $(3 \times 1)$ vector. The pose of the end-effector related to the $i-t h$ kinematic chain, $\mathbf{P}_{i}$, is defined as:

$$
\mathbf{P}_{i}=\prod_{j=1}^{n_{j, f}} \mathbf{S}_{i, j}
$$

However, the pose of the end-effector considering the joint clearances, $\mathbf{P}_{i}^{\prime}$, will not be equal to the pose $\mathbf{P}_{i}$ presented in Eq. (3). The adjoint map transformation matrix of $\mathbf{S}_{i, j}$ maps the error screw onto the end effector at a specific pose as presented in Eq. (4).

$$
\operatorname{adj}\left(\mathbf{S}_{i, j}\right)=\left[\begin{array}{cc}
\mathbf{R}_{i, j} & \mathbf{0}_{3 \times 3} \\
\mathbf{T}_{i, j} \mathbf{R}_{i, j} & \mathbf{R}_{i, j}
\end{array}\right]
$$

where $\mathbf{T}_{i, j}$ is the screw matrix of the vector $\mathbf{t}_{i, j} ; \mathbf{t}_{i, j}$ and $\mathbf{R}_{i, j}$ can be extracted from the transformation matrix of Eq. (2). Moreover, The adjoint of the inverse transformation matrix, $\operatorname{adj}\left(\mathbf{S}_{i, j}\right)^{-1}$, permits to express screws at the frame $\mathbf{F}_{i, j+1}$ from $\mathbf{F}_{i, j}$.

The error screw, $\delta \mathbf{e}_{i, j}$, in the local frame $\mathbf{F}_{i, j}$, can be expressed in the end-effector frame, $\mathbf{F}_{i, n_{i, f}}$, by multiplying all the inverse of the inverse adjoint transformation matrices from $n_{i, f}$ to $j+1$, thus: $\left(\prod_{k=n_{i, f}}^{j+1} \operatorname{adj}\left(\mathbf{S}_{i, k}\right)^{-1}\right) \delta \mathbf{e}_{i, j}$.

The following expression quantifies the pose error of the end-effector considering all the joint clearances:

$$
\delta \mathbf{p}_{i} \mid \mathbf{F}_{i, P}=\sum_{j=1}^{n_{i}} \prod_{k=n_{i, f}}^{j+1} \operatorname{adj}\left(\mathbf{S}_{i, k}\right)^{-1} \delta \mathbf{e}_{i, j}
$$

with $n_{i}$ being the number of joints, and $n_{i, f}$ the number of frames; note that $n_{i, f} \geq n_{i} . \delta_{\mathbf{P}_{i}} \mid \mathbf{F}_{i, P}$ is the pose error in the frame attached to the end-effector $\mathbf{F}_{i, P}$.

The pose error in the end-effector should be expressed in the reference frame attached to the fixed base $F_{i, 1}$. Thus,

$$
\delta \mathbf{p}_{i}\left|\mathbf{F}_{i, 1}=\prod_{j=1}^{n_{i, f}}\left(\mathbf{N}_{i, j}\right) \delta \mathbf{p}_{i}\right| F_{i, P}
$$

where $\mathbf{N}_{i, j}=\left[\begin{array}{cc}\mathbf{R}_{i, j} & \mathbf{0}_{3 \times 3} \\ \mathbf{0}_{3 \times 3} & \mathbf{R}_{i, j}\end{array}\right]$. Therefore, an expression for $\delta \mathbf{p}_{i} \mid \mathbf{F}_{i, 1}$ is obtained by substituting Eq. (5) into Eq. (6).

$$
\delta \mathbf{p}_{i} \mid \mathbf{F}_{i, 1}=\sum_{j=1}^{n_{i}} \prod_{l=1}^{n_{i, f}}\left(\mathbf{N}_{i, l}\right) \prod_{k=n_{i, f}}^{j+1} \operatorname{adj}\left(\mathbf{S}_{i, k}\right)^{-1} \delta \mathbf{e}_{i, j}
$$

The expression of Eq. (7) can be written in the following compact form:

$$
\delta \mathbf{p}=\mathbf{M}_{i} \delta \mathbf{e}_{i}
$$

where $\mathbf{M}_{i}=\left[\mathbf{M}_{i, 1} \ldots \mathbf{M}_{i, n i}\right], \delta \mathbf{e}_{i}=\left[\delta \mathbf{e}_{i, 1}^{T} \ldots \delta \mathbf{e}_{i, n_{i}}^{T}\right]$, and

$$
\mathbf{M}_{i, j}=\prod_{l=1}^{n_{i, f}}\left(\mathbf{N}_{i, l}\right) \prod_{k=n_{i, f}}^{j+1}\left(\operatorname{adj}\left(\mathbf{S}_{i, k}\right)^{-1}\right)
$$




\section{B. Parallel Mechanism}

Parallel manipulators are composed of several and identical kinematic chains that connect a fixed basis to a movable platform. Differently of serial manipulators, the parallel manipulators are subject to kinematic constraints introduced by their closed-loop kinematic configurations. These kinematic constraints must be considered to propagate the errors of the joint clearance onto the end-effector. Moreover, the pose obtained from any kinematic chain should be equal to each other, therefore $\mathbf{P}_{1}=\mathbf{P}_{2} \cdots=\mathbf{P}_{m}$.

For the model of the joint clearances, the external load that acts on the end-effector produces the errors in the joint clearance of the kinematic chains. The errors of the joints are correlated due to the kinematic constraints of the parallel mechanism, as shown in Fig. 2.

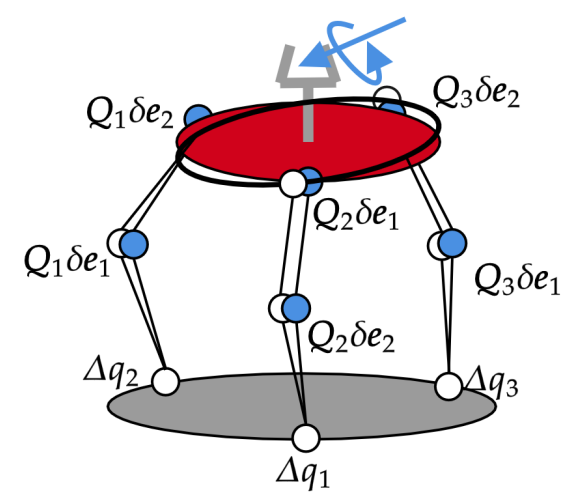

Fig. 2. Model of a parallel mechanism subjected to clearances.

The following assumptions are considered:

1) The errors of all the joint clearances of each kinematic chain defined by $\delta \mathbf{e}_{i}$ are correlated. This correlation depends on the orientation of the kinematic chains, $\mathbf{R}_{i}$, concerning the fixed frame. Thus, $\delta \mathbf{e}_{i}=$ $\mathbf{Q}_{i}\left[\delta \mathbf{e}_{i, 1}^{T} \ldots \delta \mathbf{e}_{i, n_{i}}^{T}\right]$ with $\mathbf{Q}_{i, j}=\left[\begin{array}{cc}\mathbf{R}_{i} & \mathbf{0}_{3 \times 3} \\ \mathbf{0}_{3 \times 3} & \mathbf{R}_{i}\end{array}\right]$.

2) The passive joints are free. Therefore no clearance around the axial axis is considered for the passive joints, thus $\Delta \beta_{i, j, z}=0$.

3) The errors of the prismatic or revolute active joints are entirely independent. They are defined by $\Delta \mathbf{q}=$ $\left[\Delta q_{1} \ldots \Delta q_{m}\right]^{T}$.

The errors of the joint clearances are propagated for every single kinematic chain by using the expression of Eq. (7), and the error produced by the active joints is also considered by using the Jacobian matrix $\mathbf{J}$.

$$
\delta \mathbf{p}_{i}=\mathbf{W} \mathbf{J} \delta \Delta \mathbf{q}+\mathbf{M}_{i} \delta \mathbf{e}_{i}
$$

where $\mathbf{W}$ transforms the end-effector error to an error screw. The definition of this matrix will depend on the kinematics of the parallel mechanism. The minor error along each Cartesian coordinate of the errors of every kinematic chain $\delta \mathbf{p}_{i}$ is considered in order to respect the kinematic constraints of the parallel mechanism $\left(\mathbf{P}_{1}=\mathbf{P}_{2} \cdots=\mathbf{P}_{m}\right)$. Thus, the total error in the end effector of the parallel mechanism $\delta \mathbf{p}$ is defined by the following expression.

$$
\delta \mathbf{p}=\min \left(\begin{array}{llll}
\delta \mathbf{p}_{1} & \delta \mathbf{p}_{2} & \ldots & \delta \mathbf{p}_{m}
\end{array}\right)
$$

\section{Kinematic Reliability Method}

This method aims at computing a reliability index to assess the kinematic accuracy of a mechanism subject to an uncertain error produced by the joint clearances. The proposed approach is based on the fuzzy uncertainty theory. The position error is evaluated by using Eq. (10) considering the translational error such as presented in Eq. (1). Thus, $e=\sqrt{\delta p_{4}^{2}+\delta p_{5}^{2}+\delta p_{6}^{2}}$. The uncertain parameters of the clearances are defined as fuzzy variables; thus: $\Delta \tilde{\beta}_{i, j, x y}, \tilde{\gamma}_{i}$, $\Delta \tilde{b}_{i, j, x y}$, and $\Delta \tilde{b}_{i, j, z}$. Therefore, the position error will also be a fuzzy uncertain variable.

The uncertain fuzzy error $\tilde{e}$ is defined by using the $\alpha$-level representation:

$$
\tilde{\mathbf{e}}=\left\{(e, \mu(e)) \mid e \in \mathbb{R}_{>0}\right\} \quad \text { where } 0 \leq \mu(e) \leq 1
$$

Moreover, the fuzzy error $\tilde{e}$ can be represented by continuous intervals so-called $\alpha$-levels, thus $e_{\alpha_{k}}=\{e \in$ $\left.\mathbb{R}_{>0}, \mu(e) \geq \alpha_{k}\right\}$. Alternatively, $e_{\alpha_{k}}$ can be defined as an interval weighted by the membership function $\mu(e)$, thus:

$$
e_{\alpha_{k}}=\left(0, e_{\alpha_{k} r}\right)
$$

where $e_{\alpha_{k} r}=\max \left(e \in \mathbb{R}_{>0}, \mu(e) \geq \alpha_{k}\right)$. The evaluation of $e_{\alpha_{k} r}$ demands the solution of an optimization problem to determine the upper limit of the uncertain error corresponding to the $\alpha_{k}$ value of the $\mu(e)$ membership function.

The reliability of a system is evaluated based on the limit state function $g=r-s$, where $r$ is the resistance limit, and $s$ is the system output [5]. The failure is produced when the system output exceeds the resistance limits, i.e. $g<0$. Regarding the kinematic reliability with uncertain fuzzy errors, the limit state function is defined as the $\tilde{g}$ fuzzy function, thus $\tilde{\mathbf{g}}=e_{\max }-\tilde{\mathbf{e}}$ where $e_{\max }$ is the maximum admissible position error. Fig. 3(a) illustrates the fuzzy limit state function.

The fuzzy kinematic reliability aims at determining the possibility of failure by examining the fuzzy limit state function. Figure 3(b) shows that a failure is produced when $\tilde{e}>e_{\max }$ i.e., $\tilde{g}>0$. For this condition, the possibility of failure is given by $\alpha_{f}$.

The membership function of $\tilde{\mathbf{g}}$ is approximated as a linear function in order to estimate the failure possibility considering the maximum position error $e_{\alpha 0}$ only. The following expression computes the linear estimation of the failure possibility:

$$
\alpha_{f}=\frac{e_{0}}{e_{\max }}
$$

with $e_{0}=e_{\max }-e_{\alpha_{0}}$. The uncertain fuzzy error $\tilde{\mathbf{e}}$ for $\alpha_{k}=0$ is obtained by solving the following maximization:

$$
e_{\alpha_{0}}=\max _{\boldsymbol{\lambda}} e(\boldsymbol{\lambda})
$$

with $\boldsymbol{\lambda}=\left[\Delta \beta_{1, x y} \gamma_{1} \Delta b_{1, x y} \Delta b_{i, z} \ldots \Delta \beta_{n i, x y} \gamma_{n i} \Delta b_{n i, x y} \Delta b_{n i, z}\right.$

$$
\left.\Delta q_{1} \ldots \Delta q_{m}\right]
$$




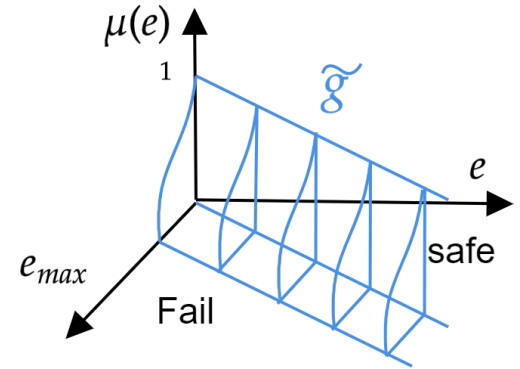

(a)

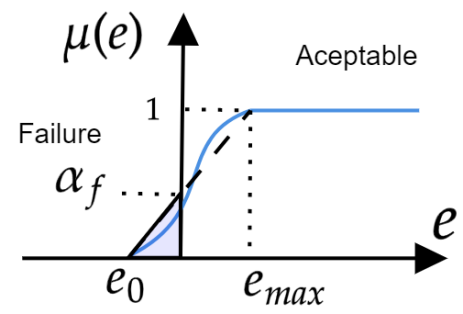

(b)

Fig. 3. Fuzzy limit state function: (a) $\tilde{\mathbf{e}}$ and $e_{\max }$ and (b) evaluation of ẽ.

It is worth to mention that the optimization problem of Eq. (14) will be solved by using the Differential Evolution algorithm (DE) [17].

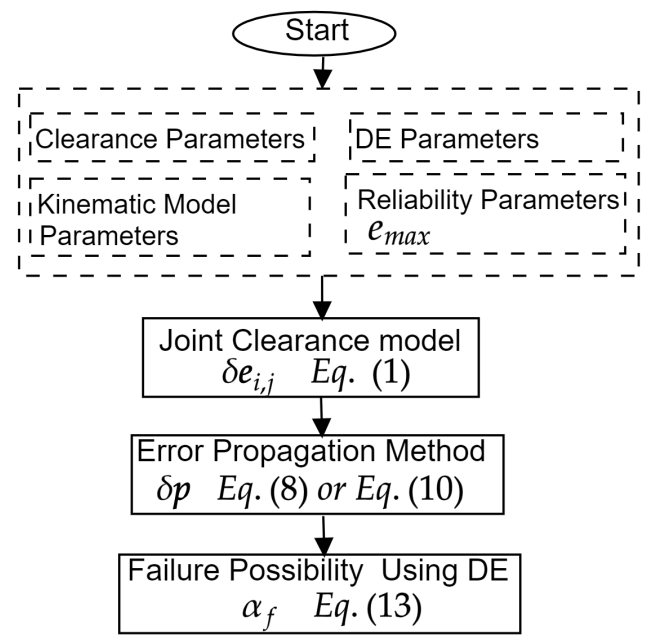

Fig. 4. Flowchart of the fuzzy kinematic approach.

The flowchart of Fig. 4 illustrates the proposed approach to compute the fuzzy kinematic reliability. This approach has three main stages: $i$ ) the clearances of the joints with fuzzy uncertainties are computed; $i i$ ) the positioning error at the end-effector is computed based on the error propagation method; iii) the fuzzy kinematic reliability is assessed based on the possibility of error definition that requires the solution of the optimization problem of Eq. (14) by using DE.

\section{Kinematic Reliability of The CPM}

The Cartesian Parallel Manipulator (CPM) has three symmetric kinematic chains that joint the moving platform $P$ to the fixed frame (see Fig. 5). The three active prismatic joints ( $\mathbf{q}=\left[q_{1} q_{2} q_{3}\right]^{T}$ ) act along the $X, Y$, and $Z$ axes. The moving platform has three translational degrees of freedom defined by $(x, y, z)$. Every kinematic chain is located at the frame $O_{j}$, and it has three passive rotational joints defined by $\theta_{j, i}$, for $i=1,2,3$, and $j=1,2,3$. The link's lengths of every kinematic chain are defined by $l_{1}, l_{2}$, and the geometry of the moving platform is defined by $l_{p}$. The active joints specify the Cartesian position of the moving platform directly, thus $q_{1}=x, q_{2}=y$, and $q_{3}=z$. Therefore, the Jacobian matrix is a $3 \times 3$ identity matrix, $\mathbf{J}=\mathbb{I}_{3 \times 3}$.

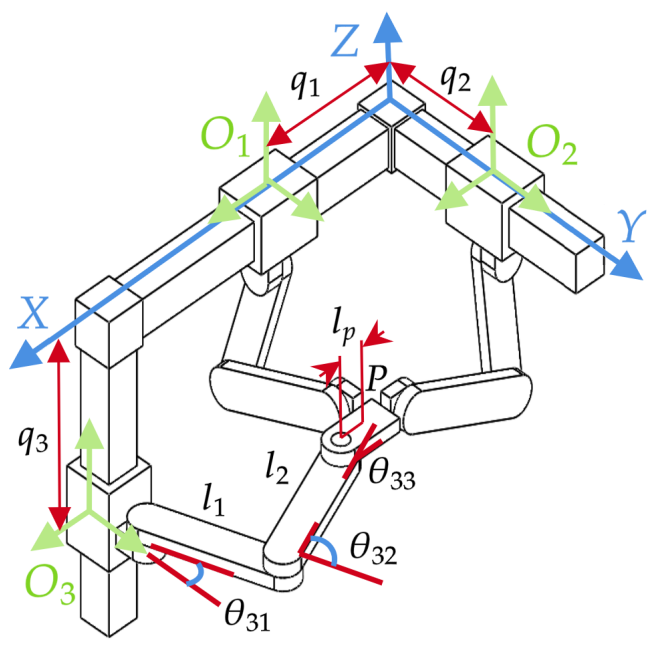

Fig. 5. Cartesian Parallel Manipulator (CPM).

The D-H parameters of the $j-t h$ kinematic chain from the frame $O_{j}$ to $P$ are presented as follows, frame $i: \alpha_{j, i-1}$, $a_{j, i-1}, d_{j, i}, \theta_{j, i}$. Thus, frame $1: 0,0,0, \theta_{j, 1} ;$ frame $2: 0, l_{1}$, $0, \theta_{j, 2}$; frame $3: 0, l_{2}, 0, \theta_{j, 3}$; frame $P: 0, l_{p}, 0,0$.

For this numerical application the geometric parameters of the manipulator and the clearances were defined as follows. The link's length was defined specifically as $l_{1}=0.077 \mathrm{~m}$, $l_{2}=0.077 \mathrm{~m}$ and $l_{p}=0.022 \mathrm{~m}$. A fuzzy triangular position error was considered for every active joint $\Delta \tilde{q}_{j}=<0,0,1 \times$ $10^{-4}>\mathrm{m}$. The uncertain parameters of the passive rotational joints clearances were as $\Delta \tilde{\beta}_{i, j, x y}=<0,0,0.002>^{o}$, $\tilde{\gamma}_{i}=<0,180,360>^{o}, \Delta \tilde{b}_{i, j, x y}=<0,0,1 \times 10^{-4}>\mathrm{m}$ and $\Delta \tilde{b}_{i, j, z}=<0,0,1 \times 10^{-4}>\mathrm{m}$. Moreover, the maximum admissible error is defined as $e_{\max }=2.5 \times 10^{-4} \mathrm{~m}$. The orientation of the kinematic chains $\mathbf{Q}_{i}$ is defined by the orientation matrices: $\mathbf{R}_{1}=\mathbb{I}_{3 x 3}, \mathbf{R}_{2}=\mathbf{R}\left(90^{\circ}, X\right)$ and $\mathbf{R}_{3}=\mathbf{R}\left(90^{\circ}, Y\right)$. According to Eq. (9), the position error is

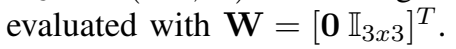

The optimization problem of the Eq. (14) was solved by using the Differential Evolution (DE) algorithm; the parameters selected to run the DE algorithm were: population size is 5 per uncertain variable, 100 generations, crossover probability rate is 0.8 , perturbation rate is 0.8 and the $D E /$ rand/1/bin strategy for the mutation mechanism. 


\section{A. Single Serial Chain}

Initially, the kinematic reliability of a single kinematic chain is analyzed separately from the Cartesian parallel manipulator; specifically, the first kinematic chain is considered in this analysis. The kinematic reliability is evaluated over the $z x$ plane for $y=0$ by using the proposed fuzzy approach (see Fig. 6(a)) and the MC method (see Fig. 6(b)).

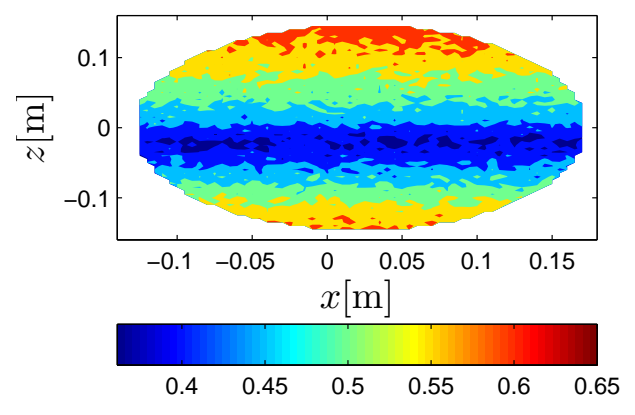

(a)

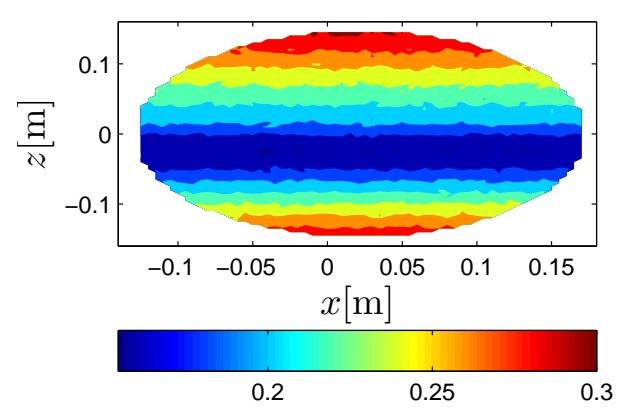

(b)

Fig. 6. Kinematic reliability of a single kinematic chain: (a) possibility of failure, (b) probability of failure.

It can be observed that the estimated kinematic reliability has a similar behavior by using the MC method and the fuzzy approach (see Fig. 6), i.e. the failure probability and failure possibility increase and decrease in the same regions of the workspace. Nevertheless, the failure possibility is related to a measure that indicates the amplitude of the positioning error, i.e., the possibility of failure quantifies the ratio of the positioning error and the maximum admissible error $e_{\max }$.

\section{B. Parallel Mechanism}

The position error of $P, \delta \mathbf{p}(\theta)$, at the end-effector position $\left[\begin{array}{lll}0.069 & 0.089 & 0.089\end{array}\right] \mathrm{m}$. Figure 7 (a) presents the fuzzy limit state function $\tilde{g}$. The failure possibility $\alpha_{f}$ estimated with the linear approximation of Eq. (13) is 0.4185 and evaluated $\tilde{g}$ is 0.358 (see Fig. 7(a)); the percentage difference between these results is about $14.4 \%$; therefore, the linear approximation of $\alpha_{f}$ is valid. The obtained $\alpha_{f}$ means $41.8 \%$ of the possibility that position error exceeds the maximum limit.

Moreover, the kinematic reliability was also evaluated using the Monte Carlo simulation as presented in Fig. 7(b). Thus, the failure probability, $p_{f}=n_{f} / n_{s}$ where $n_{f}$ is the

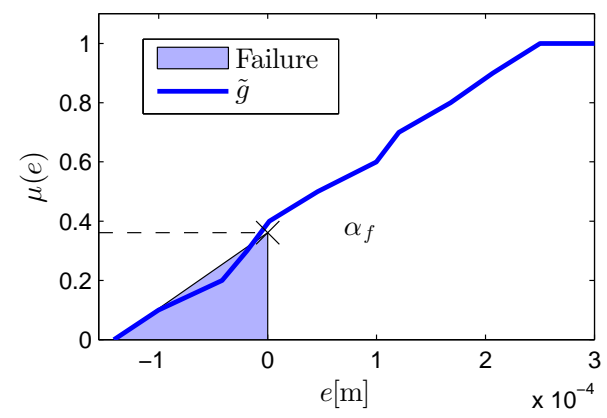

(a)

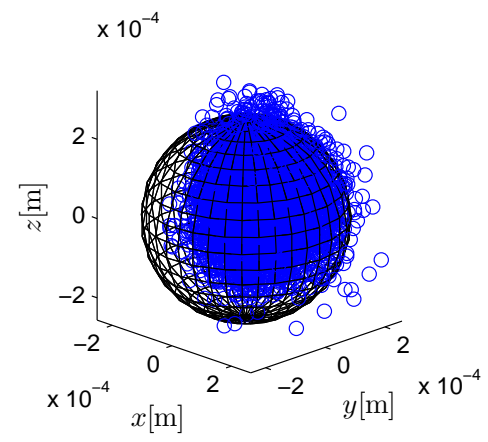

(b)

Fig. 7. Position error of $P$ : (a) $\tilde{\mathbf{g}}$, and (b) reliability analysis using MCS.

number of samples exceeding $e_{\max }$, and $n_{s}$ is the total number of samples; thus, $p_{f}=0.045$.

The kinematic reliability was estimated over a plane of the workspace as shown in Fig.8 in which $z=0$. The possibility of failure $\alpha_{f}$ is presented in Fig. 8(a). $\alpha_{f}$ increases for the regions of workspace in which the kinematic chains are extended, i.e., the extension of kinematic chain increases position error and $\alpha_{f}$. Moreover, one can observe that the possibility of failure has similar behavior with the probability of failure estimated with the MCS (see Fig. 8(b)), i.e., the possibility and probability of failure increase and decrease in the same regions of the workspace.

\section{CONCLUSIONS}

The proposed fuzzy reliability method permitted to compute the kinematic failure possibility of a cartesian parallel manipulator as an alternative to the probabilistic approaches widely used in the literature. Moreover, the proposed approach allows quantifying a kinematic reliability index that takes into account the kinematic constraints and the effect of clearances on the kinematic chains. Classical kinematic criteria based on the condition number of the Jacobian matrix will not reveal the insight behavior of the CMP in terms of the kinematic accuracy assessed by fuzzy kinematic reliability. Future work will encompass the optimal design of the mechanism based on the proposed fuzzy kinematic reliability method.

\section{ACKNOWLEDGMENT}

The authors are thankful for the financial support provided by CNPq (Process 427204/2018-6), and CAPES. 


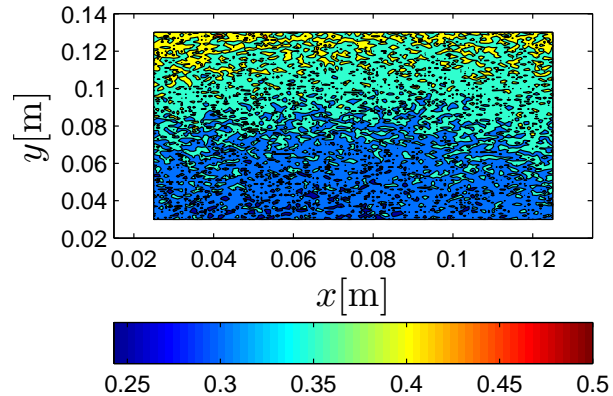

(a)

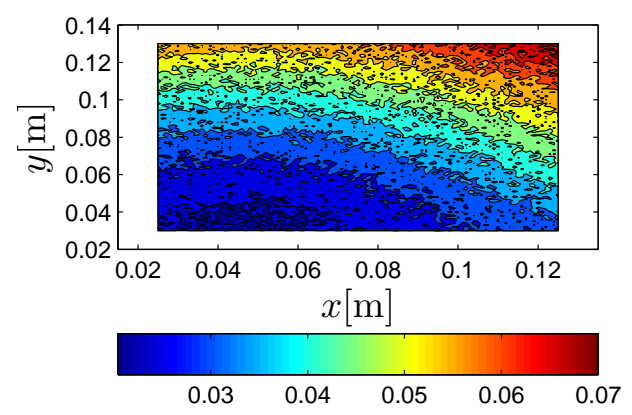

(b)
[12] X. Wang, X. Geng, L. Wang, R. Wang, X. Chen, and W. Fan, "Timedependent reliability-based dimensional synthesis for planar linkages with unknown but bounded joint clearances," Journal of Mechanical Design, vol. 140, no. 6, p. 061402, 2018.

[13] F. A. Lara-Molina, D. Dumur, and K. A. Takano, "Multi-objective optimal design of flexible-joint parallel robot," Engineering Computations, 2018.

[14] S.-J. Huang and J.-S. Lee, "A stable self-organizing fuzzy controller for robotic motion control," IEEE Transactions on Industrial Electronics, vol. 47, no. 2, pp. 421-428, 2000.

[15] L. Wang, T. Chai, and L. Zhai, "Neural-network-based terminal sliding-mode control of robotic manipulators including actuator dynamics," IEEE Transactions on Industrial Electronics, vol. 56, no. 9, pp. 3296-3304, 2009.

[16] F. Lara-Molina, K. Takano, and E. Koroishi, "Set-point regulation of a robot manipulator with flexible joints using fuzzy control," in 2015 12th Latin American Robotics Symposium and 2015 3rd Brazilian Symposium on Robotics (LARS-SBR), pp. 103-108, IEEE, 2015.

[17] R. Storn and K. Price, "Differential evolution-a simple and efficient heuristic for global optimization over continuous spaces," Journal of global optimization, vol. 11, no. 4, pp. 341-359, 1997.
Fig. 8. Kinematic reliability: (a) possibility of failure, (b) probability of failure.

\section{REFERENCES}

[1] J. Takahashi, T. Fukukawa, and T. Fukuda, "Passive alignment principle for robotic assembly between a ring and a shaft with extremely narrow clearance," IEEE/ASME Trans. on Mechatronics, vol. 21, no. 1, pp. 196-204, 2016.

[2] A.-H. Chebbi, Z. Affi, and L. Romdhane, "Prediction of the pose errors produced by joints clearance for a 3-UPU parallel robot," Mechanism and Machine Theory, vol. 44, no. 9, pp. 1768-1783, 2009.

[3] P. Voglewede and I. Ebert-Uphoff, "Application of workspace generation techniques to determine the unconstrained motion of parallel manipulators," Journal of Mech. Design, vol. 126, no. 2, pp. 283-290, 2004.

[4] J. Meng, D. Zhang, and Z. Li, "Accuracy analysis of parallel manipulators with joint clearance," Journal of Mech. Design, vol. 131, no. 1, p. 011013, 2009.

[5] M. D. Pandey and f. Zhang, "System reliability analysis of the robotic manipulator with random joint clearances," Mechanism and Machine Theory, vol. 58, pp. 137-152, 2012.

[6] F. Lara-Molina, E. Koroishi, D. Dumur, and V. Steffen Jr, "Stochastic analysis of a 6-DOF fully parallel robot under uncertain parameters," IFAC-PapersOnLine, vol. 48, no. 19, pp. 214-219, 2015.

[7] J. Zhang and X. Du, "Time-dependent reliability analysis for function generation mechanisms with random joint clearances," Mechanism and Machine Theory, vol. 92, pp. 184-199, 2015.

[8] F. Lara-Molina, E. Koroishi, V. Steffen, and L. Martins, "Kinematic performance of planar 5R symmetrical parallel mechanism subjected to clearances and uncertainties," Journal of the Brazilian Society of Mechanical Sciences and Engineering, vol. 40, no. 4, p. 189, 2018.

[9] S. Venanzi and V. Parenti-Castelli, "A new technique for clearance influence analysis in spatial mechanisms," Journal of Mechanical Design, vol. 127, no. 3, pp. 446-455, 2005.

[10] D. Xu, "Kinematic reliability and sensitivity analysis of the modified delta parallel mechanism," International Journal of Advanced Robotic Systems, vol. 15, no. 1, p. 1729881418759106, 2018.

[11] X. Geng, X. Wang, L. Wang, and R. Wang, "Non-probabilistic timedependent kinematic reliability assessment for function generation mechanisms with joint clearances," Mechanism and Machine Theory, vol. 104, pp. 202-221, 2016. 